\title{
Article \\ Univalence Conditions for Gaussian Hypergeometric Function Involving Differential Inequalities
}

\author{
Georgia Irina Oros
}

check for updates

Citation: Oros, G.I. Univalence Conditions for Gaussian Hypergeometric Function Involving Differential Inequalities. Symmetry 2021, 13, 904. https://doi.org/ $10.3390 /$ sym 13050904

Academic Editor: Ioan Rașa

Received: 16 April 2021

Accepted: 17 May 2021

Published: 19 May 2021

Publisher's Note: MDPI stays neutral with regard to jurisdictional claims in published maps and institutional affiliations.

Copyright: (C) 2021 by the author. Licensee MDPI, Basel, Switzerland. This article is an open access article distributed under the terms and conditions of the Creative Commons Attribution (CC BY) license (https:// creativecommons.org/licenses/by/ $4.0 /)$.
Department of Mathematics and Computer Science, University of Oradea, 410087 Oradea, Romania; goros@uoradea.ro

Abstract: In their paper published in 1990, Miller and Mocanu have investigated the special function Gaussian hypergeometric function in view of its relation to the theory of analytic functions, stating conditions for this function to be univalent using $a, b, c \in \mathbb{R}, c \neq 0,-1,-2, \ldots$. The study done in this paper extends the results on the univalence of the considered function taking $a, b, c \in \mathbb{C}$, with $c \neq 0,-1,-2, \ldots$ two criteria being stated in the corollaries of the proved theorems. An interpretation of the univalence results from the sets inclusion view is also given, underlining the geometrical properties of the outcomes. Examples showing how the univalence results can be applied are also included.

Keywords: differential subordination; Gaussian hypergeometric function; differential inequality in $\mathbb{C}$

\section{Introduction}

Hypergeometric functions began to be studied more intensely in terms of their relation to the field of complex analysis after proving useful in demonstrating Bieberbach's conjecture in 1985 [1]. Before that, the interest on hypergeometric functions came from their applications in different branches of mathematics such as representation theory, algebraic geometry and Hodge theory, number theory, mirror symmetry. The Gaussian hypergeometric function presented high interest, with studies concerning its applications in complex analysis being done as soon as this relation between domains was considered. One of the first papers which contained new results due to this study appeared in 1990 [2] when Miller and Mocanu considered Gaussian and confluent (Kummer) hypergeometric functions and stated some conditions for their univalence. Gaussian hypergeometric function was investigated in terms of close-to-convexity properties in [3], and some results related to its univalence were obtained before 1990 [4], but it has already been established that they were different from the ones contained in [2].More recently, results on the univalence conditions for the Gaussian hypergeometric function were given in [5-7], but in this paper the new results related to the univalence of this function are stated using the well-known theory of differential subordination introduced in two papers in 1978 [8] and 1981 [9] by Miller and Mocanu, further developed in the coming years by many authors and synthesized in the work published in 2000 [10] by the authors who introduced the notion.

Miller and Mocanu's paper published in 1990 [2] first served as inspiration for obtaining some new results related to the confluent (Kummer) hypergeometric function stated by first using notions concerning differential subordination theory [11] and then notions of differential superordiantion theory [12], with the relation between those results and the original results of Miller and Mocanu being emphasized. The study done to obtain the results contained in this paper follows the same pattern as in paper [11], but now it takes the Gaussian hypergeometric function into consideration.

All the classical definitions of notions and established notations for the special classes of univalent functions are used throughout the paper.

Let $U=\{z \in \mathbb{C}:|z|<1\}$ and let $H(U)$ denote the class of analytic functions in the unit disc. The class $H[a, n]$ is seen as the subclass of analytic functions having the particular 
form $f(z)=a+a_{n} z^{n}+a_{n+1} z^{n+1}+\cdots, z \in U$ with $a \in \mathbb{C}$ and $n \in \mathbb{N}, n \neq 0$. Another important subclass of $H(U)$ which the study refers to consists of functions written as $f(z)=z+a_{n+1} z^{n+1}+\cdots, z \in U$, this class being denoted by $A_{n}$, with $A_{1}=A$. All functions from class $A$ having the property $\operatorname{Re} \frac{\mathrm{zf}^{\prime}(\mathrm{z})}{\mathrm{f}(\mathrm{z})}>0$ are called starlike and their class is denoted by $S^{*}$, while functions from the same class with the property $\operatorname{Re} \frac{\mathrm{zf} \mathrm{f}^{\prime \prime}(\mathrm{z})}{\mathrm{f}^{\prime}(\mathrm{z})}+1>0$ are called convex and their class is denoted by $K$.

Definitions for the relation of subordination, solution of the differential subordination, dominant and best dominant for a differential subordination are well-known and can be seen in [11] (Definition 2 and Definition 3) and in Miller and Mocanu's monograph, [10] (p.4) and they are used just as they are given there.

The proofs of the original theorems from the next part of this paper require the use of a lemma which can be found in [10] (Th. 3.4h, p.132).

Lemma 1. [10] Let $q$ be univalent in $U$ and let $\theta$ and $\phi$ be analytic in a domain D containing $q(U)$, with $\phi(w) \neq 0$, when $w \in q(U)$. Set $Q(z)=z q^{\prime} \cdot \phi[q(z)]$,

$h(z)=\theta[q(z)]+Q(z)$, and suppose that either

(i) $h$ in convex, or

(ii) $Q$ is starlike.

In addition, assume that

(iii) $\operatorname{Re} \frac{z h^{\prime}(z)}{Q(z)}=\operatorname{Re}\left[\frac{\theta^{\prime}[q(z)]}{\phi[q(z)]}+\frac{z Q^{\prime}(z)}{Q(z)}\right]>0$.

If $p$ is analytic in $U$, with $p(0)=q(0), p(U) D$ and

$$
\theta[p(z)]+z p^{\prime}(z) \cdot \phi[p(z)] \prec \theta[q(z)]+z q^{\prime}(z)^{\prime} \cdot[q(z)]=h(z)
$$

then

$$
p \prec q
$$

and $q$ is the best dominant.

The notion of differential subordination was introduced after observing certain inequalities and inclusions of sets valid in real analysis and adapting them for the case of complex-valued functions. A short history of the emergence of the notion can be read in the introduction of paper [12]. In the same cited paper, some geometrical interpretations of superordination results written as inclusions of sets are shown. Such a perspective on using set inclusions to express a new outcome was approached in another paper published in 2020 [13], which proves that this direction is of interest at this time and generates an interesting outcome. This technique is applied for the original results obtained in this paper for differential subordinations.

The definition of the Gaussian hypergeometric function is reminded in [10] and presented below:

Definition 1. [10] Let $a, b, c \in \mathbb{C}, c \neq 0,-1,-2, \ldots$ The function:

$$
F(a, b, c ; z)={ }_{2} F_{1}(a, b, c ; z)=1+\frac{a b}{c} \cdot \frac{z}{1 !}+\frac{a(a+1) b(b+1)}{c(c+1)} \cdot \frac{z^{2}}{2 !}+\cdots, z \in U,
$$

is called a Gaussian hypergeometric function.

A property easily obtained for this function given in [2] is:

$$
c \cdot\left[{ }_{2} F_{1}(a, b, c ; z)\right]^{\prime}=a b \cdot{ }_{2} F_{1}(a+1, b+1, c+1 ; z)
$$


Next to the mirror symmetry formula for Gauss hypergeometric function, it is known that this function has the symmetric property remaining unaltered if the numerator parameters $a$ and $b$ are interchange while, keeping the denominator parameter $c$ fixed.

The original results obtained in relation to this function and which have led to stating two criteria for its univalence can be seen in the following theorems and corollaries.

\section{Main Results}

Using Lemma 1 mentioned in the introduction, the first stated theorem proves a subordination result which, for certain specific functions used, gives criteria for the univalence of the Gaussian hypergeometric function. The criteria are presented in two corollaries that follow the theorem, and the geometrical interpretation of the results in the corollaries using the inclusion of sets can also be seen.

Theorem 1. Consider a convex function $q$ having the property $\operatorname{Re} q(z)>0, z \in U$ and let $\varphi \in H(D)$ when $q(U) \subset D$, knowing that $\varphi(\omega) \neq 0$ for $\omega \in q(U)$.

Let $\theta: \mathbb{C} \rightarrow \mathbb{C}$ and $\psi: \mathbb{C}^{2} \rightarrow \mathbb{C}$. Take:

$$
Q(z)=z \cdot q^{\prime}(z) \cdot \varphi[q(z)] \text { and } h(z)=Q(z)+\theta[q(z)] .
$$

Let $p \in H(U)$ with the condition $p(0)=q(0)$ be met when $p(U) \subset D$.

If the subordination:

$$
p^{2}(z)+z p^{\prime}(z) \prec h(z),
$$

is satisfied, then $p(z) \prec q(z)$ when $z \in U$, written as the sets inclusion $p(U) \subset q(U)$, and $q$ is the best dominant.

Proof. The proof uses Lemma 1, and hence the conditions from the lemma must be met. To prove the compliance of the first condition from the lemma, the functions $\theta: \mathbb{C} \rightarrow \mathbb{C}$, $\varphi: \mathbb{C} \rightarrow \mathbb{C}$ and $\psi: \mathbb{C}^{2} \rightarrow \mathbb{C}$ are defined as:

$$
\theta(\omega)=\omega^{2}, \varphi(\omega)=1, \psi(r, s)=r^{2}+s, r, s \in \mathbb{C} .
$$

Putting $\omega=q(z)$ in (5), the following equalities are obtained: $z q^{\prime}(z)$.

$\theta[q(z)]=q^{2}(z), \varphi[q(z)]=1$ and $r=q(z), s=z q^{\prime}(z), \psi\left(q(z), z q^{\prime}(z)\right)=q^{2}(z)+$

Using (3), it results in:

$$
\begin{gathered}
Q(z)=z \cdot q^{\prime}(z) \cdot 1 \\
h(z)=q^{2}(z)+z q^{\prime}(z) \cdot 1, z \in U,
\end{gathered}
$$

and:

$$
\psi\left(q(z), z q^{\prime}(z)\right)=\theta[q(z)]+z q^{\prime}(z) \cdot \varphi[q(z)]=q^{2}(z)+z q^{\prime}(z), z \in U .
$$

Putting $\omega=p(z)$ in (5), the following equalities are obtained: $z p^{\prime}(z)$.

$\theta[p(z)]=p^{2}(z), \varphi[p(z)]=1$ and $r=p(z), s=z p^{\prime}(z), \psi\left(p(z), z p^{\prime}(z)\right)=p^{2}(z)+$

Using (3) again, it results in:

$$
\psi\left(p(z), z p^{\prime}(z)\right)=\theta[p(z)]+z p^{\prime}(z) \cdot \varphi[p(z)]=p^{2}(z)+z p^{\prime}(z), z \in U .
$$

Using (8) and (9), the differential subordination (4) becomes:

$$
[q(z)]+z q^{\prime}(z) \cdot \varphi[q(z)] \prec \theta[p(z)]+z p^{\prime}(z) \cdot \varphi[p(z)] .
$$


To apply Lemma 1, the starlikeness of function $Q$ needs to be proved. In the hypothesis of the theorem, function $q$ was taken as convex. Thus, it is known that:

$$
\operatorname{Re}\left(\frac{z q^{\prime \prime}(z)}{q^{\prime}(z)}+1\right)>0, z \in U
$$

Differentiating (6) and making a simple calculus, we obtain:

$$
\frac{z q^{\prime}(z)}{Q(z)}=1+\frac{z q^{\prime \prime}(z)}{q^{\prime}(z)}, z \in U
$$

Using (11) in (12), we have:

$$
\operatorname{Re} \frac{z q^{\prime}(z)}{Q(z)}=\operatorname{Re}\left[1+\frac{z q^{\prime \prime}(z)}{q^{\prime}(z)}\right]>0, z \in U
$$

which is equivalent to $Q$ being starlike. It is now proved that the first condition needed in order to apply Lemma 1 is satisfied.

Differentiating (7) and after a simple calculus, using (11) and the hypothesis of the theorem, we get:

$$
\operatorname{Re} \frac{z h^{\prime}(z)}{Q(z)}=2 \operatorname{Req}(z)+\operatorname{Re}\left[1+\frac{z q^{\prime \prime}(z)}{q^{\prime}(z)}\right]>0, z \in U,
$$

which means that the second condition from Lemma 1 is also satisfied.

Using Lemma 1, the differential subordination (10) implies:

$$
p(z) \prec q(z)
$$

when $z \in U$.

Since $q$ is a convex function which satisfies the equation $h(z)=q^{2}(z)+z q^{\prime}(z), z \in U$ it is the best dominant of the proved differential subordination.

Remark 1. If in Theorem 1, the function:

$$
q(z)=\frac{1+z}{1-z}, q(0)=1, z \in U,
$$

is considered since it is a convex function, and if $p(z)={ }_{2} F_{1}(a+1, b+1, c+1 ; z)$ given by (1), then the following univalence criterion for the Gaussian hypergeometric function is obtained.

Corollary 1. Consider the convex function:

$$
q(z)=\frac{1+z}{1-z}, q(0)=1, z \in U
$$

and let $\varphi \in H(D)$ when $q(U) \subset D$, knowing that $\varphi(\omega) \neq 0$ for $\omega \in q(U)$.

Take:

$$
Q(z)=z \cdot q^{\prime}(z) \cdot 1=\frac{2 z}{(1-z)^{2}}
$$

and:

$$
\begin{gathered}
h(z)=\frac{1+4 z+z^{2}}{(1-z)^{2}} \\
\text { Let } p(z)={ }_{2} F_{1}(a+1, b+1, c+1 ; z) \in H(U), p(z)={ }_{2} F_{1} \text { given by }(1) \text { with } \\
p(0)={ }_{2} F_{1}(a+1, b+1, c+1 ; 0)=q(0)=1
\end{gathered}
$$

when $p(U) \subset D$. 


\section{If the subordination:}

$$
\left[{ }_{2} F_{1}(a+1, b+1, c+1 ; z)\right]^{2}+z \cdot\left[{ }_{2} F_{1}(a+1, b+1, c+1 ; z)\right]^{\prime} \prec h(z)=\frac{1+4 z+z^{2}}{(1-z)^{2}}
$$

is satisfied, then:

$$
p(z)={ }_{2} F_{1}(a+1, b+1, c+1 ; z) \prec \frac{1+z}{1-z}
$$

when $z \in U$, written as the sets inclusion:

$$
p={ }_{2} F_{1}(U) \subset\{z \in \mathbb{C}: z=x+i y, x>0, y \in \mathbb{R}\},
$$

i.e.,

$$
\operatorname{Re} \frac{c}{a b} \cdot\left[{ }_{2} F_{1}(a, b, c ; z)\right]^{\prime}>0
$$

written as the sets inclusion:

$$
\frac{c}{a b} \cdot\left[{ }_{2} F_{1}\right]^{\prime}(U) \subset\{z \in \mathbb{C}: z=x+i y, x>0, y \in \mathbb{R}\},
$$

Hence, $p(z)={ }_{2} F_{1}(a+1, b+1, c+1 ; z)$ is univalent in $U$.

Proof. Let:

$$
\begin{gathered}
p(z)={ }_{2} F_{1}(a+1, b+1, c+1 ; z)=1+\frac{(a+1)(b+1)}{c+1} \cdot \frac{z}{1 !}+\frac{(a+1)(a+2)(b+1)(b+2)}{(c+1)(c+2)} \cdot \frac{z^{2}}{2 !}+\cdots, z \in U, \\
p(0)=1, p \in H[1,1] .
\end{gathered}
$$

Next, we differentiate the equality and we obtain:

$$
p^{\prime}(z)=\left[{ }_{2} F_{1}(a+1, b+1, c+1 ; z)\right]^{\prime}, z \in U .
$$

Then, the differential subordination (15) becomes:

$$
[p(z)]^{2}+z p^{\prime}(z) \prec \frac{1+4 z+z^{2}}{(1-z)^{2}}, z \in U .
$$

Using relation (14) from the proof of Theorem 1 results in:

$$
p(z) \prec q(z)=\frac{1+z}{1-z}
$$

and:

$$
p(z)={ }_{2} F_{1}(a+1, b+1, c+1 ; z) \prec q(z)=\frac{1+z}{1-z}
$$

Using (2), we obtain:

$$
\frac{c}{a b} \cdot\left[{ }_{2} F_{1}(a, b, c ; z)\right]^{\prime \prime}={ }_{2} F_{1}(a+1, b+1, c+1 ; z), z \in U .
$$

Using (17) in (16), we have:

$$
\frac{c}{a b} \cdot\left[{ }_{2} F_{1}(a, b, c ; z)\right]^{\prime \prime} \prec q(z)=\frac{1+z}{1-z}, z \in U .
$$

Using the convexity of function $q(z)$, the differential subordination (18) is equivalent to:

$$
\operatorname{Re} \frac{c}{a b} \cdot\left[{ }_{2} F_{1}(a, b, c ; z)\right]^{\prime}>\operatorname{Re} \frac{1+z}{1-z}>0, z \in U,
$$


written as the sets inclusion:

$$
\frac{c}{a b} \cdot\left[{ }_{2} F_{1}\right]^{\prime}(U) \subset\{z \in \mathbb{C}: z=x+i y, x>0, y \in \mathbb{R}\},
$$

concluding that the function $p(z)={ }_{2} F_{1}(a+1, b+1, c+1 ; z)$ is univalent.

Remark 2. In Theorem 1, take the functions:

$$
p(z)=\frac{z\left[{ }_{2} F_{1}(a, b, c ; z)\right]^{\prime \prime}}{\left[{ }_{2} F_{1}(a, b, c ; z)\right]^{\prime}}, q(z)=\frac{1+z}{1-z}, z \in U
$$

The following univalence criterion for the Gaussian hypergeometric function can be written:

Corollary 2. Consider the convex function:

$$
q(z)=\frac{1+z}{1-z}, q(0)=1, z \in U
$$

and let $\varphi \in H(D)$ when $q(U) \subset D$, knowing that $\varphi(\omega) \neq 0$ for $\omega \in q(U)$.

Take:

$$
Q(z)=z \cdot q^{\prime}(z)=\frac{2 z}{(1-z)^{2}}
$$

and:

$$
h(z)=\frac{1+4 z+z^{2}}{(1-z)^{2}}
$$

If the function:

$$
1+\frac{z\left[{ }_{2} F_{1}(a, b, c ; z)\right]^{\prime \prime}}{\left[{ }_{2} F_{1}(a, b, c ; z)\right]^{\prime}}, a, b, c \in \mathbb{C}, c \neq 0,-1,-2, \ldots
$$

is analytic in $U$ and satisfies the differential subordination:

$$
\left\{1+\frac{z\left[{ }_{2} F_{1}(a, b, c ; z)\right]^{\prime \prime}}{\left[{ }_{2} F_{1}(a, b, c ; z)\right]^{\prime}}\right\}^{2}+z \cdot\left\{1+\frac{z\left[{ }_{2} F_{1}(a, b, c ; z)\right]^{\prime \prime}}{\left[{ }_{2} F_{1}(a, b, c ; z)\right]^{\prime}}\right\}^{\prime} \prec \frac{1+4 z+z^{2}}{(1-z)^{2}}
$$

Then:

$$
1+\frac{z\left[{ }_{2} F_{1}(a, b, c ; z)\right]^{\prime \prime}}{\left[{ }_{2} F_{1}(a, b, c ; z)\right]^{\prime}} \prec q(z)=\frac{1+z}{1-z}, z \in U,
$$

i.e.,

$$
\operatorname{Re}\left\{1+\frac{z\left[{ }_{2} F_{1}(a, b, c ; z)\right]^{\prime \prime}}{\left[{ }_{2} F_{1}(a, b, c ; z)\right]^{\prime}}\right\}>0
$$

written as the sets inclusion:

$$
p={ }_{2} F_{1}(U) \subset\{z \in \mathbb{C}: z=x+i y, x>0, y \in \mathbb{R}\},
$$

Hence, the function $F(a, b, c ; z)={ }_{2} F_{1}(a, b, c ; z)$ is convex in $U$.

Proof. Let:

$$
p(z)=1+\frac{z\left[{ }_{2} F_{1}(a, b, c ; z)\right]^{\prime \prime}}{\left[{ }_{2} F_{1}(a, b, c ; z)\right]^{\prime}}=1+p_{1} z+p_{2} z^{2}+\cdots, p(0)=1, p \in H[1,1] .
$$


Differentiating the equality and using the expressions for $p(z)$ and $p^{\prime}(z)$ in (19), we get:

$$
[p(z)]^{2}+z p^{\prime}(z) \prec \frac{1+4 z+z^{2}}{(1-z)^{2}}, z \in U .
$$

Using relation (14) obtained in the proof of Theorem 1 results in:

$$
p(z) \prec q(z)=\frac{1+z}{1-z}
$$

By replacing $p(z)$ with its expression written above, we obtain:

$$
1+\frac{z\left[{ }_{2} F_{1}(a, b, c ; z)\right]^{\prime \prime}}{\left[{ }_{2} F_{1}(a, b, c ; z)\right]^{\prime}} \prec q(z)=\frac{1+z}{1-z}, z \in U,
$$

and function $q(z)$ is the best dominant.

Using the convexity of function $q(z)$, the above differential subordination is equivalent to:

$$
\operatorname{Re}\left\{1+\frac{z\left[{ }_{2} F_{1}(a, b, c ; z)\right]^{\prime \prime}}{\left[{ }_{2} F_{1}(a, b, c ; z)\right]^{\prime}}\right\}>\operatorname{Re} \frac{1+z}{1-z}>0, z \in U,
$$

written as the sets inclusion:

$$
p(U) \subset\{z \in \mathbb{C}: z=x+i y, x>0, y \in \mathbb{R}\},
$$

concluding that function $F(a, b, c ; z)={ }_{2} F_{1}(a, b, c ; z)$ is convex in $U$.

This corollary is a key result as it gives the connection between the original outcome of this paper and the findings of Miller and Mocanu in their paper [2] which have inspired this study, and it is also used in the proof of the next two original theorems.

Remark 3. The outcome proved in Corollary 2 has been previously obtained in [2] having considered $a, b, c \in \mathbb{R}$, with the properties $-2<a<0,-1 \leq b, b \neq 0, c>M(a, b)$ where $M(a, b)=$ $\max \{2+|a+b|, 1-a b\}$.

The next theorem gives results concerning the starlikeness of the derivative of the Gaussian hypergeometric function. The proof of this theorem is short because it uses Corollary 2.

Theorem 2. Let $F(a, b, c ; z)={ }_{2} F_{1}(a, b, c ; z)$ given by (1). Then, $z \cdot \frac{c}{a b} \cdot\left[{ }_{2} F_{1}(a, b, c ; z)\right]^{\prime}$ is starlike.

Proof. Let $a b \neq 0$,

$$
\begin{gathered}
g(z) \quad z \cdot \frac{c}{a b} \cdot\left[{ }_{2} F_{1}(a, b, c ; z)\right]^{\prime} \\
=z \cdot \frac{c}{a b} \cdot\left(1+\frac{a b}{c} \cdot \frac{z}{1 !}+\frac{a(a+1) b(b+1)}{c(c+1)} \cdot \frac{z^{2}}{2 !}+\cdots\right)^{\prime} \\
=z+p_{2} z^{2}+\cdots, g(0)=0, g \in A, g^{\prime}(z)=1 \neq 0 .
\end{gathered}
$$

Differentiating and doing some simple calculus, the following equality is obtained:

$$
\frac{z g^{\prime}(z)}{g(z)}=1+\frac{z\left[{ }_{2} F_{1}(a, b, c ; z)\right]^{\prime \prime}}{\left[{ }_{2} F_{1}(a, b, c ; z)\right]^{\prime}}, z \in U .
$$
hence:

Corollary 2 states that function $F(a, b, c ; z)={ }_{2} F_{1}(a, b, c ; z)$ is a convex function in $U$,

$$
\operatorname{Re} \frac{z g^{\prime}(z)}{g(z)}=\operatorname{Re}\left\{1+\frac{z\left[{ }_{2} F_{1}(a, b, c ; z)\right]^{\prime \prime}}{\left[{ }_{2} F_{1}(a, b, c ; z)\right]^{\prime}}\right\}>0, z \in U,
$$

which means that function $g$ is starlike, i.e., $z \cdot \frac{c}{a b} \cdot\left[{ }_{2} F_{1}(a, b, c ; z)\right]^{\prime}$ is starlike. 
The next theorem states a starlikeness result for the Gaussian hypergeometric function and also invokes Corollary 2 in the proof. The theorem makes another connection with the results from [2].

Theorem 3. Let $F(a, b, c ; z)={ }_{2} F_{1}(a, b, c ; z)$ given by (1). Then, $z \cdot{ }_{2} F_{1}(a+1, b+1, c+1 ; z)$ is starlike in $U$.

Proof. From (2), it is known that:

$$
c \cdot\left[{ }_{2} F_{1}(a, b, c ; z)\right]^{\prime}=a b \cdot{ }_{2} F_{1}(a+1, b+1, c+1 ; z)
$$

which is equivalent to:

$$
z \cdot \frac{c}{a b} \cdot\left[{ }_{2} F_{1}(a, b, c ; z)\right]^{\prime}=z \cdot{ }_{2} F_{1}(a+1, b+1, c+1 ; z) .
$$

Define the function $g(z)$ as:

$$
\begin{gathered}
g(z)=z \cdot \frac{c}{a b} \cdot\left[{ }_{2} F_{1}(a, b, c ; z)\right]^{\prime} \\
=z \cdot \frac{c}{a b} \cdot\left(1+\frac{a b}{c} \cdot \frac{z}{1 !}+\frac{a(a+1) b(b+1)}{c(c+1)} \cdot \frac{z^{2}}{2 !}+\cdots\right)^{\prime} \\
=z+\frac{(a+1)(b+1)}{c+1} \cdot z^{2}+\cdots
\end{gathered}
$$

having $g(0)=0, g \in h[0,1], g^{\prime}(0)=1 \neq 0$.

Differentiating $g(z)$, we obtain:

$$
\frac{z g^{\prime}(z)}{g(z)}=1+\frac{z\left[{ }_{2} F_{1}(a, b, c ; z)\right]^{\prime \prime}}{\left[{ }_{2} F_{1}(a, b, c ; z)\right]^{\prime}}, z \in U .
$$

Using Corollary 2, it is known that function $F(a, b, c ; z)={ }_{2} F_{1}(a, b, c ; z)$ is convex in $U$, which gives:

$$
\operatorname{Re} \frac{z g^{\prime}(z)}{g(z)}=\operatorname{Re}\left\{1+\frac{z\left[{ }_{2} F_{1}(a, b, c ; z)\right]^{\prime \prime}}{\left[{ }_{2} F_{1}(a, b, c ; z)\right]^{\prime}}\right\}>0, z \in U,
$$

meaning that the function $\mathrm{g}$ is starlike in $U$.

However, the function $g(z)$ was defined as $z \cdot 2 F_{1}(a+1, b+1, c+1 ; z)$, and hence the proof is concluded.

Remark 4. The starlikeness result of this theorem was previously given in [2] taking $a, b, c \in \mathbb{R}$, with the properties $-1<a<1,0 \leq b, c>1+M(a-1, b-1)$ where $M(a, b)=\max \{2+|a+b|, 1-a b\}$.

The study presented is concluded with two examples illustrating the way to apply the theoretical findings presented in the paper. The first example refers to this last proved result and the second is related to Corollary 2.

Example 1. Take $a=-1, b=2+2 i, c=2-2 i$.

Then:

$$
\begin{aligned}
F(a, b, c ; z) & ={ }_{2} F_{1}(a, b, c ; z)={ }_{2} F_{1}(-2,3+3 i, 3-3 i ; z)=1+\frac{(-1)(2+2 i)}{2-2 i} \cdot \frac{z}{1 !} \\
& =1+\frac{(-1)(1+1 i)}{1-1 i} \cdot \frac{z}{1 !}=1-\frac{(1+i)^{2}}{2} \cdot z \\
& =1-\frac{(1+2 i-1)}{2} \cdot z=\frac{2-2 i}{2} \cdot z=1-i z .
\end{aligned}
$$


Define the function $g(z)=z \cdot{ }_{2} F_{1}(-1,2+2 i, 2-2 i ; z)=z-i z^{2}$. We next prove that Theorem 3 can be applied for this function, meaning that $g(z)$ is a starlike function.

$$
\begin{aligned}
g^{\prime}(z) & =1-2 i z \Rightarrow \operatorname{Re} \frac{z g^{\prime}(z)}{g(z)}=\operatorname{Re} \frac{z(1-2 i z)}{z(1-i z)}=\operatorname{Re} \frac{1-2 i(\cos \alpha+i \sin \alpha)}{1-i(\cos \alpha+i \sin \alpha)} \\
& =\operatorname{Re} \frac{1-2 i \cos \alpha+2 \sin \alpha}{1+\sin \alpha-i \cos \alpha}=\operatorname{Re} \frac{(1+2 \sin \alpha-2 i \cos \alpha)(1+\sin \alpha+i \cos \alpha)}{(1-\sin \alpha)^{2}+\cos ^{2} \alpha} \\
& =\operatorname{Re} \frac{(1+2 \sin \alpha)(1+\sin \alpha)+2 \cos ^{2} \alpha+i[\cos \alpha(1+2 \sin \alpha)-2 \cos \alpha(1+\sin \alpha)]}{1+2 \sin \alpha+\sin ^{2} \alpha+\cos ^{2} \alpha} \\
& =\frac{1+2 \sin \alpha+\sin \alpha+2 \sin ^{2} \alpha+2 \cos ^{2} \alpha}{2(1+\sin \alpha)}=\frac{3(1+\sin \alpha)}{2(1+\sin \alpha)}=\frac{3}{2}
\end{aligned}
$$

Since:

$$
\operatorname{Re} \frac{z g^{\prime}(z)}{g(z)}>0
$$

We conclude that function $g(z)=z \cdot{ }_{2} F_{1}(-1,2+2 i, 2-2 i ; z)$ is starlike and:

$$
g(U) \subset\left\{z \in \mathbb{C}: z=x+i y, x>\frac{3}{2}, y \in \mathbb{R}\right\} .
$$

Example 2. Take $a=-2, b=i, c=-i$. Then, $F(-2, i,-1 ; z)={ }_{2} F_{1}(-2, i,-1 ; z),\left[{ }_{2} F_{1}(-2, i\right.$, $-1 ; z)]^{\prime}=2-2 i z,\left[{ }_{2} F_{1}(-2, i,-1 ; z)\right]^{\prime \prime}=-2 i$ and:

$$
1+\frac{z\left[2{ }_{2} F_{1}(-2, i,-1 ; z)\right]^{\prime \prime}}{\left[{ }_{2} F_{1}(-2, i,-1 ; z)\right]^{\prime}}=\frac{1-2 i z}{1-i z} .
$$

Take:

$$
q(z)=\frac{1+z}{1-z}, q(0)=1, z \in U
$$

and denote:

$$
p(z)=1+\frac{z\left[2 F_{1}(-2, i,-1 ; z)\right]^{\prime \prime}}{\left[{ }_{2} F_{1}(-2, i,-1 ; z)\right]^{\prime}}=\frac{1-2 i z}{1-i z} .
$$

Differentiating, we get:

$$
p^{\prime}(z)=\frac{-i}{(1-i z)^{2}}
$$

and:

$$
p^{2}(z)+z p^{\prime}(z)=\left(\frac{1-2 i z}{1-i z}\right)^{2}-\frac{i z}{(1-i z)^{2}} .
$$

Using Corollary 2, we obtain:

If:

$$
\left(\frac{1-2 i z}{1-i z}\right)^{2}-\frac{i z}{(1-i z)^{2}} \prec \frac{1+4 z+z^{2}}{(1-z)^{2}}=h(z)
$$

Then:

$$
p(z)=1+\frac{z\left[{ }_{2} F_{1}(-2, i,-1 ; z)\right]^{\prime \prime}}{\left[{ }_{2} F_{1}(-2, i,-1 ; z)\right]^{\prime}}=\frac{1-2 i z}{1-i z} \prec \frac{1+z}{1-z}, z \in U,
$$

written as the sets inclusion:

$$
p(U) \subset\{z \in \mathbb{C}: z=x+i y, x>0, y \in \mathbb{R}\},
$$

hence, $F(-2, i,-1 ; z)={ }_{2} F_{1}(-2, i,-1 ; z)$ is a convex function.

Indeed:

$$
\begin{aligned}
\operatorname{Re}\left\{1+\frac{\left.z\left[2 F_{1}(a, b, c ; z)\right]\right]^{\prime \prime}}{\left[{ }_{2} F_{1}(a, b, c ; z)\right]^{\prime}}\right\} & =\operatorname{Re} \frac{1-2 i z}{1-i z}=\operatorname{Re} \frac{1+2 \sin \alpha-2 i \cos \alpha}{1+\sin \alpha-i \cos \alpha} \\
& =\frac{3(1+\sin \alpha)}{2(1+\sin \alpha)}=\frac{3}{2}>0,
\end{aligned}
$$


$z \in U$, which shows that $F(-2, i,-1 ; z)=2 F_{1}(-2, i,-1 ; z)$ is convex in $U$, written as the sets inclusion:

$$
F(-2, i,-1 ; z)={ }_{2} F_{1}(-2, i,-1 ; z)(U) \subset\{z \in \mathbb{C}: z=x+i y, x>0, y \in \mathbb{R}\} .
$$

\section{Conclusions}

The original theorems and corollaries of this paper are the outcome of a study done on the Gaussian hypergeometric function using the theory of differential subordination in order to obtain conditions for its univalence. The results are extensions of previously obtained results, with the connection being emphasized in some remarks. The first theorem has as corollaries two criteria for univalence, for which the geometrical interpretation with sets inclusion is also given. The second corollary is a key result used in the proofs of the next two theorems and connects the results presented here with those that have inspired this study. The third theorem gives further details about this connection. The examples are enclosed in order to give an idea about the use of the theoretical results presented in the paper, and they could serve as inspiration for further studies done using specific functions. The results of this paper could inspire similar studies on this function considering the dual theory of differential superordination. A connection between the outcome of that study and the results in this paper could be sought in order to achieve the statement of sandwich type theorems, familiar to the Geometric Theory of Analytic Functions.

Funding: This research received no external funding.

Institutional Review Board Statement: Not applicable.

Informed Consent Statement: Not applicable.

Data Availability Statement: Not applicable.

Conflicts of Interest: The author declares no conflict of interest.

\section{References}

1. De Branges, L. A proof of the Bieberbach conjecture. Acta Math. 1985, 154, 137-152. [CrossRef]

2. Miller, S.S.; Mocanu, P.T. Univalence of Gaussian and confluent hypergeometric functions. Proc. Am. Math. Soc. 1990, 110, 333-342. [CrossRef]

3. Ponnusamy, S. Close-to-convexity properties of Gaussian hypergeometric functions. J. Comput. Appl. Math. 1998, 88, 327-337. [CrossRef]

4. Ruscheweyh, S.; Singh, V. On the order of starlikeness of hypergeometric functions. J. Math. Anal. App. 1986, 113, 1-11. [CrossRef]

5. Ponnusamy, S.; Vuorinen, M. Univalence and convexity properties for Gaussian hypergeometric functions. Rocky Mt. J. Math. 2001, 31, 327-353. [CrossRef]

6. Ponnusamy, S.; Vuorinen, M. Starlikeness of the Gaussian hypergeometric functions. Complex Var. Elliptic Equ. 2010, 55, 173-184. [CrossRef]

7. Swaminathan, A. Certain sufficiency conditions on Gaussian hypergeometric functions. J. Inequal. Pure App. Math. $2004,5,83$.

8. Miller, S.S.; Mocanu, P.T. Second order differential inequalities in the complex plane. J. Math. Anal. Appl. 1978, 65, 289-305. [CrossRef]

9. Miller, S.S.; Mocanu, P.T. Differential subordinations and univalent functions. Mich. Math. J. 1981, 28, 157-172. [CrossRef]

10. Miller, S.S.; Mocanu, P.T. Differential Subordinations. Theory and Applications; Marcel Dekker, Inc.: New York, NY, USA; Basel, Switzerland, 2000.

11. Oros, G.I. New Conditions for Univalence of Confluent Hypergeometric Function. Symmetry 2021, 13, 82. [CrossRef]

12. Oros, G.I. Applications of Inequalities in the Complex Plane Associated with Confluent Hypergeometric Function. Symmetry 2021, 13, 259. [CrossRef]

13. Antonino, J.A.; Miller, S.S. Systems of simultaneous differential inequalities, inclusions and subordinations in the complex plane. Anal. Math. Phys. 2020, 10, 32. [CrossRef] 